\title{
A citation-based, author- and age-normalized, logarithmic index for evaluation of individual researchers independently of publication counts [version 1; peer review: 2 approved]
}

\author{
Aleksey V. Belikov¹, Vitaly V. Belikov² \\ ${ }^{1}$ Otto-von-Guericke-Universität, Magdeburg, Germany \\ ${ }^{2}$ Water Problems Institute, Russian Academy of Sciences, Moscow, Russian Federation
}

V1 First published: 22 Sep 2015, 4:884

https://doi.org/10.12688/f1000research.7070.1

Latest published: 22 Sep 2015, 4:884

https://doi.org/10.12688/f1000research.7070.1

\section{Abstract}

The use of citation metrics for evaluation of individual researchers has dramatically increased over the last decade. However, currently existing indices either are based on misleading premises or are cumbersome to implement. This leads to poor assessment of researchers and creates dangerous trends in science, such as overproduction of low quality articles. Here we propose an index (namely, the L-index) that does not depend on the number of publications, accounts for different co-author contributions and age of publications, and scales from 0.0 to 9.9. Moreover, it can be calculated with the help of freely available software.

\section{Keywords}

Research , scientific, metrics, impact, weighted, bibliometric , scientometric, Publish or Perish

Open Peer Review
Approval Status
and Technology Information, Daejeon, South
Korea
2. Danielle M. Colbert-Lewis, North Carolina
Central University, Durham, USA
Any reports and responses or comments on the
article can be found at the end of the article.

Corresponding author: Aleksey V. Belikov (belikov.research@gmail.com)

Competing interests: The authors are active researchers and hence are affected by current research evaluation practices. The authors have no professional or personal connections to Harzing.com, Google Scholar or any other citation database or citation analysis software developers.

Grant information: The author(s) declared that no grants were involved in supporting this work.

Copyright: (c) 2015 Belikov AV and Belikov VV. This is an open access article distributed under the terms of the Creative Commons Attribution License, which permits unrestricted use, distribution, and reproduction in any medium, provided the original work is properly cited.

How to cite this article: Belikov AV and Belikov VV. A citation-based, author- and age-normalized, logarithmic index for evaluation of individual researchers independently of publication counts [version 1; peer review: 2 approved] F1000Research 2015, 4:884 https://doi.org/10.12688/f1000research.7070.1

First published: 22 Sep 2015, 4:884 https://doi.org/10.12688/f1000research.7070.1 


\section{Introduction}

There is an ever-present need to evaluate researchers' performance, because resources are limited and contenders are numerous. Before the advent of journal impact factors (JIFs, ${ }^{1}$ ), all evaluations were performed via peer review. Although JIFs were intended for librarians to decide which journals to subscribe to, they have become a commonly used proxy for the quality of journal articles ${ }^{2}$. However, the distribution of citations to individual articles within a journal is highly skewed. Twenty five percent of the most highly cited articles can account for $90 \%$ of a journal's $\mathrm{IF}^{3}$. The rest of the articles receive a few citations each, if any. Thus, using JIFs for assessing the quality of individual articles and, further, for evaluating researchers is categorically not recommended ${ }^{4}$.

The rapid development of the internet and electronic citation databases, such as PubMed, Google Scholar, Web of Science, Scopus, CiteSeer and others, has made it easier to count citations of individual articles. It is now possible to automatically calculate the total number of citations that the publications of a given researcher have accumulated. However, these numbers can range from 1 to 100,000 and, obviously, do not represent the equal variation in researchers' capabilities. For example, human IQ scores vary only about $2-4$ fold $^{5}$.

There are two main reasons why the total number of citations cannot be used to adequately compare individual researchers. First, there is usually more than one author for each publication. Some of the most highly cited articles, such as reports from experiments on particle accelerators ${ }^{6}$ or from genome sequencings ${ }^{7}$, and guidelines for medical practitioners ${ }^{8}$, have from tens to hundreds of co-authors, usually listed in alphabetical order. Thus, it is inadequate to assign all the thousands of citations to each of those authors. A similar situation is when a researcher operates some very expensive and thus rare equipment, and is listed on papers of all other researchers who perform experiments on that equipment ${ }^{9}$. It is clear that the researcher also does not deserve all citations of those papers, as his contribution is purely technical. Many ways to divide citations between co-authors have been proposed ${ }^{10,11}$, but the only practical way is to split them equally, i.e. to assign each co-author $\mathbf{1} / \boldsymbol{n}$ of the citations, where $\boldsymbol{n}$ is the total number of co-authors.

Another factor is that citations accumulate with time. A researcher who started his career 30 years ago will undoubtedly have more citations than a young postdoc, but this does not necessarily mean that the former is a better scientist. Moreover, a paper that has been highly cited is likely to be cited even more in the future ${ }^{12}$. Thus, citations exhibit the behavior of preferential attachment, which results in their distribution according to the power law ${ }^{13}$. These considerations make it necessary to adjust for the age of each publication, in order to properly assess current capabilities and impact of researchers, not their past successes, and to partially compensate for the preferential attachment. Dividing the number of citations by the age of the publication in years seems to be an adequate measure, as it mirrors the power law distribution that citations have.

Finally, a large variety of individual citation metrics have been proposed $^{14}$, the most widely disseminated of which is the h-index ${ }^{15}$. The drawback of the majority of these metrics is that they take into consideration the number of publications. For example, the h-index can never exceed the total number of publications a scientist has. However, several researchers of undisputed scientific merit, such as Sir Isaac Newton, Gregor Mendel or Peter Higgs, have published only a few, however significant, works. This lack in the number of publications leads them to have h-indices of 4,1 and 9, respectively, which are disparagingly low. All other derivatives of the h-index, as well as all indices that take into account publication counts, suffer from the same drawback and hence should never be used for evaluation purposes. However, they are used, promoting a grueling and futile quest for quantity of publications, at the expense of quality, reflected in the infamous "publish or perish" catchphrase ${ }^{16}$. Fortunately, this issue has been recently called to public attention, most notably in the form of the San Francisco Declaration on Research Assessment, and some measures have been proposed ${ }^{17}$.

Overall, there is an immense need for a simple but reliable indicator for individual researcher assessment. Here, we propose such an index, which accounts for different co-author contributions and age of publications, and does not depend on the number of publications. Moreover, it conveniently ranges from 0.0 to 9.9, and can be calculated with the help of freely available software.

\section{Methods}

To address the concerns highlighted in the introduction of this article, we have set out to construct an index that accounts for different co-author contributions and age of publications. This has initially led us to the following formula:

$$
I=\sum_{i=1}^{N} \frac{c_{i}}{a_{i} y_{i}}
$$

where $I$-preliminary index, $c_{i}$ - number of citations to $i$-th publication, $a_{i}$ - number of authors of $i$-th publication, $y_{i}-$ age in years of $i$-th publication, $N$ - number of publications.

We then decided to estimate the range of values that our preliminary index can have. First, we calculated $I$ for a hypothetical $\mathrm{PhD}$ student who recently received the first citation to his first paper, with 5 authors: $I=\frac{1}{5 \times 1}=0.2$. Next, we calculated $I$ for Albert Einstein and Charles Darwin, two of the most prominent and well-known scientists. To this aim, we utilized the freely available software Publish or Perish. This program imports citation data from Google Scholar and allows removal of irrelevant results, such as publications of homonym authors. A parameter AWCRpA (age-weighted citation rate per author) can be obtained from this program, and is equivalent to $I$ from formula (1). The AWCRpA values for Einstein and Darwin, as calculated by Publish or Perish at the time of writing this article, were 6466 and 6178, respectively. Thus, even upon correcting for multiple authorship and age, citations vary by approximately 30,000 fold. As it is unlikely that the human brain can exhibit such a tremendous difference in its efficiency or talents, normalized citations should be mapped to a more appropriate scale, in order to make them more useful in meaningfully comparing researchers. It seems that a $1-10$ scale is optimal, because it is closer to the true variation in human intellectual or other capabilities $^{5}$, and is widely used in various metrics, thus being more 
intuitive. The natural logarithm function appears to be ideal for this scaling purpose. Compared to the square root or the cube root, the natural logarithm allows better resolution of differences between the majority of scientists, with the exception of the most prominent ones (Figure 1). To account for some of the negative values that arise when there is less than one normalized citation, the resulting index is increased by one point. In extreme cases where the index value still remains negative, it is advised to simply consider it as zero.

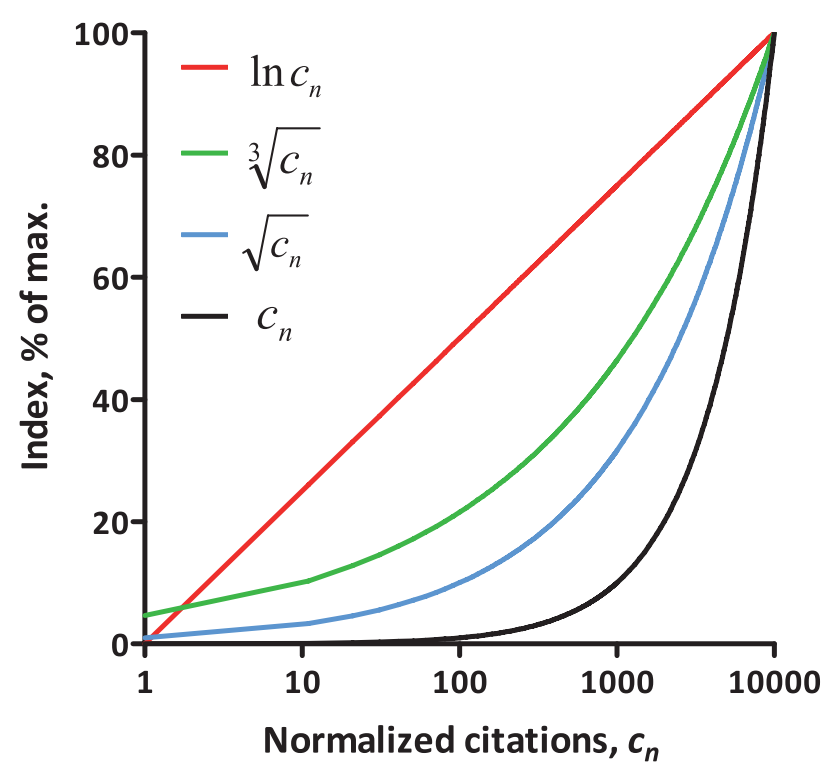

Figure 1.The natural logarithm function increases evenly across the citation range. The natural logarithm $\left(\operatorname{In} c_{n}\right)$, cube root $\left(\sqrt[3]{c_{n}}\right)$ and square root $\left(\sqrt{c_{n}}\right)$ functions of normalized citations $\left(c_{n}\right)$ are shown.
Finally, the formula for the Logarithm index (L-index) has become:

$$
L=\ln \left(\sum_{i=1}^{N} \frac{c_{i}}{a_{i} y_{i}}\right)+1
$$

where $c_{i}$ - number of citations to $i$-th publication, $a_{i}$ - number of authors of $i$-th publication, $y_{i}-$ age in years of $i$-th publication, $N$ - number of publications.

When $\sum_{i=1}^{N} \frac{c_{i}}{a_{i} y_{i}}$ is calculated as AWCRpA in Publish or Perish, formula (2) takes the form:

$$
L=\ln A W C R p A+1
$$

Equation 3 has been used to obtain all L-index values in this article.

To calculate typical L-indices for a $\mathrm{PhD}$ student, a postdoc and a principal investigator (PI), we averaged the L-index values for $5 \mathrm{PhD}$ students, 10 postdocs and 15 PIs that we personally know (see Supplementary Table 1).

\section{Results and discussion}

Figure 2 shows the typical scale of the L-index with the indication of the values for ten of some of the most prominent and widely recognized scientists, as well as the typical values for a $\mathrm{PhD}$ student, a postdoc and a principal investigator (PI) (see Supplementary Table 1).

It can be seen from this figure that the L-index adequately captures the intuitive ranking, i.e. $\mathrm{PhD}$ student $<$ Postdoc $<\mathrm{PI}<\ldots<$ Albert Einstein. Moreover, it allows the objective (or, at least, statistically averaged collective subjective) quantitative assessment of researchers, which is a virtue that traditional peer review cannot accomplish.

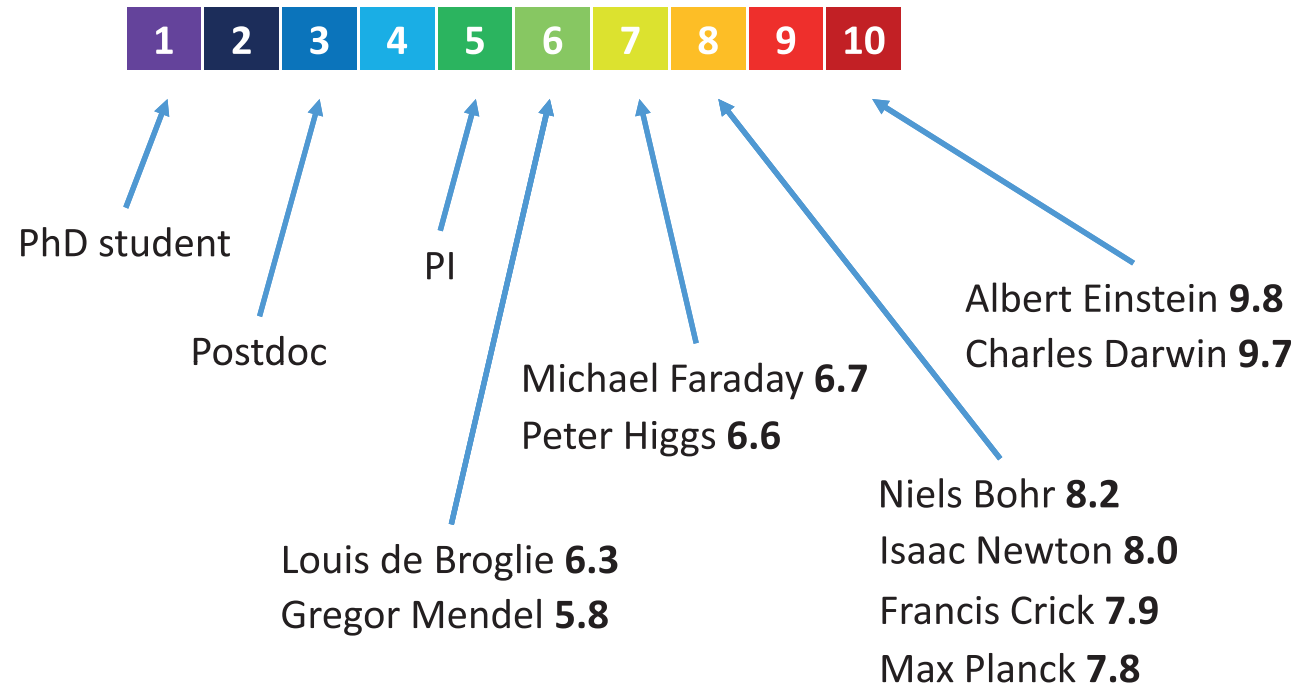

Figure 2. The L-index scale and notable examples. The typical range of the L-index is shown. The positions of 10 of the most famous scientists are indicated, along with their L-index scores. The positions of a typical PhD student, a postdoc and a principal investigator (PI) are also displayed. The raw data can be seen in Supplementary Table 1. 
However, in cases where L-indices of the applicants are equal up to one decimal place, we strongly suggest the use of peer review, involving thorough examination of their publications, rather than differentiation of scientists based on the second decimal place, to avoid false precision and statistical bias. In case of young researchers that have only a few citations, it is also advisable to use peer review, as the limited data do not allow for the statistically robust calculation of the citation index.

The L-index can increase or decrease with time, as it depends on the age of publications. Thus, it favors the impact of recent publications and gives a much needed advantage to younger researchers. However, if a scientist has made such a significant discovery that its impact only increases with time, his L-index will stay high regardless of the age of the publication. Perfect examples of this are Albert Einstein and Charles Darwin. Despite them ceasing to publish original work decades ago, their L-indices are still higher than those of the absolute majority of current researchers (Figure 2).

The quantitative comparison of the L-index with other evaluation indices, such as the h-index, is purposefully avoided in this article, for the reason that those indices have been designed on different premises, such as to account for the number of publications. When evaluating the performance of a researcher, it should first be decided which parameter is considered adequate for the purpose the number of publications, which does not tell anything about their quality, or the number of citations, which, however indirectly, indicates the impact that the publications have made. If the latter option is selected, the L-index can help to account for the effects of multiple co-authorship and aging of publications, and present the results in a simple and intuitive form.

\section{Author contributions}

AVB co-created the early version of the citation index formula, created the final L-index formula, collected and analyzed the data, interpreted the results and wrote the manuscript. VVB co-created the early version of the citation index formula, interpreted the results and revised the manuscript. All authors have agreed to the final content.

\section{Competing interests}

The authors are active researchers and hence are affected by current research evaluation practices. The authors have no professional or personal connections to Harzing.com, Google Scholar or any other citation database or citation analysis software developers.

\section{Grant information}

The authors declared that no grants were involved in supporting this work.

\section{Acknowledgements}

The authors are grateful to Prof. A. Borthwick, Dr. A. Barmashov, and V. Hrubilov for the useful discussions.

\section{Supplementary material}

Table 1. The raw data that was used to calculate $\mathbf{L}$-indices for Figure 2.

AWCRpA were obtained from Publish or Perish, upon excluding publications of homonym authors. L-indices were calculated according to formula (3), using Excel formula editor and $L N$ function. Average L-indices were calculated using AVERAGE function.

Click here to access the data.

1. Garfield E: Citation analysis as a tool in journal evaluation. Science. 1972; 178(4060): 471-9.

PubMed Abstract | Publisher Full Text

2. Adam D: The counting house. Nature. 2002; 415(6873): 726-9. PubMed Abstract | Publisher Full Text

3. Not-so-deep impact. Nature. 2005; 435(7045): 1003-4. PubMed Abstract | Publisher Full Text

4. Beware the impact factor. Nat Mater. 2013; 12(2): 89. PubMed Abstract | Publisher Full Text

5. Hunt E: Human intelligence. Cambridge University Press, 2010. Reference Source

6. Aad G, Abajyan T, Abbott B, et al:: Observation of a new particle in the search for the Standard Model Higgs boson with the ATLAS detector at the LHC.
Phys Lett B. 2012; 716(1): 1-29.

Publisher Full Text

7. Venter JC, Adams MD, Myers EW, et al:: The sequence of the human genome. Science. 2001; 291(5507): 1304-51.

PubMed Abstract | Publisher Full Text

8. Perk J, De Backer G, Gohlke H, et al.: European Guidelines on cardiovascular disease prevention in clinical practice (version 2012): The Fifth Joint Task Force of the European Society of Cardiology and Other Societies on Cardiovascular Disease Prevention in Clinical Practice (constituted by representatives of nine societies and by invited experts). Atherosclerosis. 2012; 223(1): 1-68.

PubMed Abstract | Publisher Full Text

9. Anderson C: Authorship. Writer's cramp. Nature. 1992; 355(6356): 101 PubMed Abstract | Publisher Full Text 
10. Tscharntke T, Hochberg ME, Rand TA, et al.: Author sequence and credit for contributions in multiauthored publications. PLOS Biol. 2007; 5(1): e18. PubMed Abstract | Publisher Full Text | Free Full Text

11. Shen HW, Barabási AL: Collective credit allocation in science. Proc Natl Acad Sci U S A. 2014; 111(34): 12325-30.

PubMed Abstract | Publisher Full Text | Free Full Text

12. Merton RK: The Matthew Effect in Science: The reward and communication systems of science are considered. Science. 1968; 159(3810): 56-63. PubMed Abstract | Publisher Full Text

13. Price DDS: A general theory of bibliometric and other cumulative advantage processes. J Am Soc Inf Sci. 1976; 27(5): 292-306.

Publisher Full Text
14. Van Noorden R: Metrics: A profusion of measures. Nature. 2010; 465(7300): 864-6.

PubMed Abstract | Publisher Full Text

15. Hirsch JE: An index to quantify an individual's scientific research output. Proc Natl Acad Sci U S A. 2005; 102(46): 16569-72. PubMed Abstract | Publisher Full Text | Free Full Text

16. Garfield E: What is the primordial reference for the phrase 'publish or perish'? The Scientist. 1996; 10(12): 11.

Reference Source

17. Hicks D, Wouters $\mathrm{P}$, Waltman L, et al:: Bibliometrics: The Leiden Manifesto for research metrics. Nature. 2015; 520(7548): 429-31.

PubMed Abstract | Publisher Full Text 


\section{Open Peer Review}

\section{Current Peer Review Status:}

\section{Version 1}

Reviewer Report 17 November 2015

https://doi.org/10.5256/f1000research.7610.r10836

C 2015 Colbert-Lewis D. This is an open access peer review report distributed under the terms of the Creative Commons Attribution License, which permits unrestricted use, distribution, and reproduction in any medium, provided the original work is properly cited.

\section{Danielle M. Colbert-Lewis}

Department of James E. Shepard Memorial Library, North Carolina Central University, Durham, NC, USA

This title is appropriate for the content of the article and the abstract represents a suitable summary for the work. The design, methods and analysis used in the article represented a thorough approach to the subject. The conclusions are suitable, balanced and justified on the basis of the results of the study. The data represented allows enough information to replicate the experiment for further research. The data is usable with the structure that has been provided.

Competing Interests: No competing interests were disclosed.

\section{I confirm that I have read this submission and believe that I have an appropriate level of expertise to confirm that it is of an acceptable scientific standard.}

Reviewer Report 16 October 2015

https://doi.org/10.5256/f1000research.7610.r10421

(C) 2015 Jung $\mathbf{Y}$. This is an open access peer review report distributed under the terms of the Creative Commons Attribution License, which permits unrestricted use, distribution, and reproduction in any medium, provided the original work is properly cited.

\section{Youngim Jung}

Korea Institute of Science and Technology Information, Daejeon, South Korea

This paper proposes a novel index based on the number of citations, normalized by the number of authors and the date of publication, which is then scaled from 0.0 to 9.9 using the natural logarithm. The index proposed is intuitively designed in a simple way for readers to understand, with a fractional counting scheme adopted to normalize the number of citations, which appears 
reasonable. However, it will be necessary to adjust the index according to the relationship between the number of citations and the date of publication, since they are not precisely inversely proportional in every time window.

Finally and fundamentally, whether the number of authors and the date of publication are truly indicative of publication quality remains open to speculation.

Competing Interests: No competing interests were disclosed.

I confirm that I have read this submission and believe that I have an appropriate level of expertise to confirm that it is of an acceptable scientific standard.

\section{Comments on this article}

\section{Version 1}

Author Response 30 Nov 2021

Aleksey Belikov, Otto-von-Guericke-Universität, Magdeburg, Germany

The L-index has now been calculated for 3 million scientists with public ORCID record. It is available for each year separately, and the percentile amongst all authors is shown.

The rating of all authors according to the L-index is also available: https://exaly.com/authors/lindex

Competing Interests: No competing interests were disclosed.

Reader Comment 26 Nov 2021

Niga Targhisi, University of South Carolina, USA

exaly has calculated L-Index for 3 million authors. The L-index values are accompanied by the percentile among all authors, as you can test your theory if the 0-9.9 categories really represent the academic categories you considered.

In addition, the L-index values have been reported for the previous years, as one can check the changes in L-index as the number of research publications of an author increases or his/her research works progress.

A quick look confirms your idea for the categorization of authors, but it needs a thorough investigation.

Competing Interests: No competing interests were disclosed. 


\section{Author Response 29 Aug 2016}

Aleksey Belikov, Otto-von-Guericke-Universität, Magdeburg, Germany

It would be amazing if somebody could create a simple website that would calculate this index, and possibly other indices that I've proposed, based on Google Scholar data. This way anyone could easily test their usefulness. Please contact me if you are willing to do such a site.

Competing Interests: No competing interests were disclosed.

The benefits of publishing with F1000Research:

- Your article is published within days, with no editorial bias

- You can publish traditional articles, null/negative results, case reports, data notes and more

- The peer review process is transparent and collaborative

- Your article is indexed in PubMed after passing peer review

- Dedicated customer support at every stage

For pre-submission enquiries, contact research@f1000.com 